\title{
La pedagogía como saber fundante de la formación del maestro. Algunas precisiones
}

\author{
JUAN FELIPE GARCÉS GÓMEZ*
}

\author{
AJUAN FRANCISCO \\ "Quid est veritas?... \\ -Nosotros, respondr" \\ Umberto Eco'
}

\section{El problema}

$\mathrm{P}$ reguntarse por el papel de la pedagogía como saber fundante de la formación del maestro, presupone ya la aceptación de algunos asuntos sumamente discutibles. En primer lugar, se presupone que sabemos qué es aquello que llamamos 'pedagogía' o que, al menos, hay algunos consensos sobre ella. Una rápida mirada a las recientes publicaciones en el país prueba que estamos muy lejos de lo que presuponemos, mientras algunos se aferran a la historia, otros se lanzan a conquistar los terrenos de la neurociencia o de la experiencia humana más íntima. En medio de esta situación babélica, todo el panorama se hace más oscuro si reconocemos que permanentemente la pedagogía es refundada sin ninguna dificultad desde saberes aje-

Profesor de la Universidad de Antioquia.

1. Eco, Umberto. Elpéndulo de Foucault. nos a sus indagaciones, los cuales se reivindican la fundación de ese campo de problemas y conceptos que desde los albores de la modernidad llamamos PEDAGOGía. Presuponemos, además, qué es la formación y lo que creemos es o debe ser un maestro. En fin, lo que aún puede resultarnos más complejo es que creemos saber qué es un 'Saber'.

Cabe precisar, para continuar, dos aspectos: En primer lugar no aceptamos la sedimentación y usos indiscriminados de los conceptos foucaultianossaber, discurso y práctica, entre otrossin poder dar cuenta razonablemente de los mismos en el contexto de la propuesta del autor o en las reflexiones sobre la ciencia, conocida en Francia como epistemología, en los países anglosajones como filosofia de la ciencia y en Alemania como teoría del conocimiento. En segundo lugar, no aceptamos que la pedagogía sea un 'algo' dirigido exclusivamente a la formación y desempeño profesional de los enseñantes, puesto que ello evidencia la ya clásica confusión entre pedagogía y didáctica, entendiendo erróneamente esta última como método de enseñanza. La pedagogía no concierne exclusivamen- 
te a enseñantes de alguna ciencia. Es perentorio reconocer, pues, la participación de otros sujetos en el desarrollo de la pedagogía como comunidad de indagación -entiéndase como campo intelectual de la educación (DIAZ, 1993) o pedagógico (ECHEVERRI, 1997)-, entre ellos, los especuladores o "pedagogos" dedicados a la reflexión pedagógica en sus búsquedas por consolidar un espacio autónomo pero abierto para sus indagaciones y los 'experimentadores' que se ocupan exclusivamente de la constatación de los alcances y limitaciones de las propuestas didácticas. Finalmente, no todos los 'productos' de la pedagogía son para y por los enseñantes, cabe la posibilidad de emprender indagaciones donde el maestro no esté asociado indefectiblemente al aula y los alumnos, es posible concebir una indagación exclusivamente diseñada para saber del maestro en su relación con referentes sociales, culturales, científicos e históricos. La pedagogía no puede confinar al maestro al aula o al alumno, como si ellos se constituyeran en una especie de prisión conceptual, en tanto que el maestro sería un simple reproductor de tendencias o modelos didácticos, olvidando la necesidad de formar un profesional y no un técnico con habilidades para la metodización de cualquier ciencia o de una específicamente.

Este reconocimiento de los diversos sujetos que intervienen en las indagaciones pedagógicas y didácticas, nos puede permitir afrontar la pregunta fundamental de esta contribución: ¿En quiénes debemos pensar cuando nos preguntamos por las instituciones que serán escenarios de las indagaciones pedagógicas y la formación de aquellos que continuarán con éstas en el futuro?. Ya esta pregunta instala al maestro en espacios diferentes al aula y a relaciones distintas al alumno. Sí, debería participar mucho más en la comunidad de indagación desde su formación inicial, y ello no es otra cosa que la apertura de canales de comunicación con la producción nacional e internacional, no sólo de la comunidad de indagación de la disciplina que enseñará sino de la pedagogía y la didáctica. Estos canales no pueden ser otros que los desarrollados por la unidad académica universitaria donde se lleva a cabo esta formación, claro está si ella misma participa de los 'productos' de la comunidad e indagación más amplia, de no ser así, la unidad académica universitaria -llámese como se quiera- no será en nada diferente a las instituciones donde enseñaran sus egresados. Es decir, sin una plena participación en esa comunidad de indagación más amplia, solo habrá reproducción y, no pocas veces será acrítica y sometida a modas intelectuales, por sólo citar algunas, el constructivismo en educación y lo que llama Morin 'complejidad'.

En los aportes -ya no tan recientes- de la filosofía de la ciencia, podemos hallar conceptos y problemas que iluminen el problema en que estamos sumidos en la formación inicial de los maestros: Construir esa comunidad de indagación pedagógica, con problemas y conceptos propios, con una noción común y abierta de experiencia. Si no construimos una institución formadora de maestros así, la pedagogía como otras ciencias sociales, estará sometida a la dura crítica de Hacking (1996) a las ciencias sociales:

“...muchos de nosotros experimentamos cierta nostalgia, un sentimiento de tristeza, cuando estudiamos las ciencias 
sociales. Quizá es porque le hace falta lo que es tan importante acerca de la ciencia fisica moderna. Los científicos sociales no carecen de experimentos, ni carecen de cálculos. La especulación no les falta. Lo que no tienen es la colaboración entre los tres. Tampoco colaborarán, sospecho, hasta que no encuentren entes teóricos reales acerca de los cuales puedan especular, no sólo postular "constructos" y "conceptos", sino entes que podamos usar, entes que sean parte de la creación deliberada de nuevos fenómenos estables".

En otras palabras, y ya para finalizar la formulación del problema, mientras la formación del maestro sea esencialmente contenidista y puerocentrista, separada de las discusiones de la comunidad de indagación pedagógica, no se podrá acceder a una concepción de experiencia que vehicule la colaboración anhelada por Hacking entre "especuladores", "experimentadores" y maestros. Colaboración que para el autor constituye algo así como la posibilidad de hacer de la pedagogía, como ciencia social, un saber no vergonzante y tampoco sujeto a los vaivenes de modas intelectuales.

\section{El contexto}

Desde la publicación del célebre libro de T.S. Kuhn, "La estructura de las revoluciones científicas" (1971), se apoderó de las diversas comunidades de investigadores un sentimiento profundo de debilidad ante la constatación de la historicidad de sus propias prácticas. El impacto enorme no lo causó la lla- mada "sociologización" de la ciencia sino la constatación de la historicidad de la misma. Lo particular de este impacto indiscutible de las tesis kuhnianas fue que esta historicidad de la ciencia se interpretó como signo evidente de "irracionalidad", al considerar que se rompía radicalmente con la idea clásica de una ciencia que, tanto en sus preocupaciones como en sus resultados, se creían atemporales, por no decir eternos. Esto implica, a su vez, que la comprensión de nociones como racionalidad, verdad, realidad, método, lenguaje, observación, teoría o práctica, sufrieron una seria reconfiguaración al introducir las variables de la historicidad y el desarrollo comunitario de los problemas científicos. Sin embargo, como cree Hacking (1996) la esterilidad de estas discusiones remite a ideas imprecisas más que a la situación misma de la ciencia, a saber:

“... La búsqueda de la verdad y de la razón indudablemente se organizará de acuerdo con las mismas fórmulas sociales de otras buisquedas, como la felicidad o el genocidio. El hecho de que los cientificos sean gente, y de que las sociedades cientificas sean sociedades, no tiene que arrojar dudas sobre la racionalidad cientifica".

Este remezón de la percepción de la ciencia ${ }^{2}$ se presta para serios equívocos, que sumados a una radical incomprensión del teorema de Gödel y las críticas a la ascepsia de la investigación científica en materia ético-políti$\mathrm{ca}$, indican un camino expedito hacia la validez de casi cualquier cosa que sea

2. Ciencia con mayúscula para aludir a aquella tradición del pensamiento que identifica Los desarrollos de la geometría, matemática o fisica con la ciencia misma y las erigen como modelos de toda investigación posible, como criterios de "racionalidad" de cualquier otro problema suceptible de ser abordado por la inquietud humana. 
expresable lingüísticamente. Esta postura que se apoya en la peregrina idea de "lo real como lenguaje y el lenguaje como real" -parodiando a Hegeles bien conocida como "relativismo" o "antirrealismo" en sus más diversas versiones. Sin embargo, estas interpretaciones de algunos filósofos de las ciencias -especialmente sociales o humanas- no son adecuadas y tienden a sumir a los investigadores en confusiones mayores. En opinión de Sokal (1998):

"Decir que la realidad fisica es un constructo lingüistico y social es una auténtica tontería, pero decir que la realidad social es un constructo lingüístico y social es prácticamente una tautología"

Estas posturas que en Estados Unidos se conocen como ciencia posmoderna y, entre nosotros, conocemos a través de la filosofía francesa "posestructuralista", son motivo de vivos debates en las tradiciones filosóficas donde se desarrollaron. Tales debates no suelen ser captados en su real dimensión y solemos asumir tesis para el debate como logros definitivos. A esto nos referimos como modas intelectuales que a cada paso reconfiguran la pedagogía desconociendo sin más lo acaecido antes. Esta postura acrítica impide el desarrollo y reconocimiento de una tradición que impida estos desmanes conceptuales. El caso de la física contemporánea puede brindar un ejemplo de estas novedades que no pocas veces carecen de sentido.
La comunidad de especialistas en temas culturales -como prefieren denominarse los que antaño se ocupaban de las llamadas "moral science" en norteamérica- fueron sorprendidos por una ingeniosa "broma" del físico Sokal. Tal broma argumentativa fue publicada como contribución para el número especial de Social Text dedicado a la "guerra de los métodos"3 y, allí, mientras pretende presentar algunos aspectos de su especialidad, enumera las características del "paradigma de la ciencia posmoderna" en forma paródica. Tal artículo pone en el centro mismo del debate la extrapolación abusiva de las conceptualizaciones y métodos "alternativos" para afrontar problemas de diversas especialidades. Esta extrapolación pasó inadvertida para los editores y fue recibida con alborozo por los miembros de las comunidades científicas inclinadas a nuevos paradigmas para la investigación, hasta que el mismo Sokal expuso públicamente su recurso a la parodia como una reducción al absurdo originado por el uso y abuso de ciertas exploraciones metódicas que no pocas veces se manifiestan como verdaderos galimatías. El debate, originado en esta broma, pone en evidencia la situación actual de las reflexiones en torno a lo que alguna vez se llamó "científico". ¿Si aquella broma se coló en un campo de investigaciones que durante la modernidad se ha eregido como paradigmático, qué se podrá esperar en un campo como el de la pedagogía, con sus vicisitudes y falencias, con el permanente estado de crisis que bien enuncia Echeverri (1997)?

3. SOKAL, Alan. Transgrediendo los límites: hacia una hermenéutica transformadora de la gravedad cuántica. En: La balsa de la medusa. Madrid. 1998. N $45-46$. (Apareció originalmente en noviembre 28 de 1994, revisada en 1995). 
PAIDEIA

\section{Propuesta}

Envueltos en esta balcanización metódica, la pedagogía se halla más vulnerable que nunca. Las expectativas se multiplican alrededor de la utilidad y validez de un campo de investigaciones que parece ser facilmente reemplazable por estrategias cognitivas, planificaciones económicas y políticas. Se ha de constatar que no son pocos los que sostienen públicamente la superfluidad de tal campo o lo reducen a didácticas específicas, mientras otros lo consideran mera erudición histórica o filosófica. Quizá por ello la pedagogía libra sus batallas en dos frentes, por un lado busca el reconocimiento de su existencia en el marco de otros campos de investigación $y$, por otro, trata de determinar qué le compete y cómo asumir esa competencia.
En Colombia, desde hace tres décadas, el debate sobre la pedagogía se ha centrado en hallar los elementos que permitan establecer la autonomía de ésta en relación con otros campos de investigación, entre ellos la sociología o la sicología. Tal trabajo académico, y en ocasiones gremial -a saber, el movimiento pedagógico-, permiten identificar con entera claridad un campo de investigación pedagógica y/o educativa, presentado sistemáticamente por el profesor Díaz como "campo intelectual de la educación en Colombia" (Díaz 1993). Este campo, en opinión del autor, adolece de lo que denomina "epistemismo", es decir, una preocupación por determinar el "estatuto disciplinar de la pedagogía" frente a las disciplinas hegemónicas que la reclaman para sí como uno de sus subcampos. Este "epistemismo" produce, contrario a lo

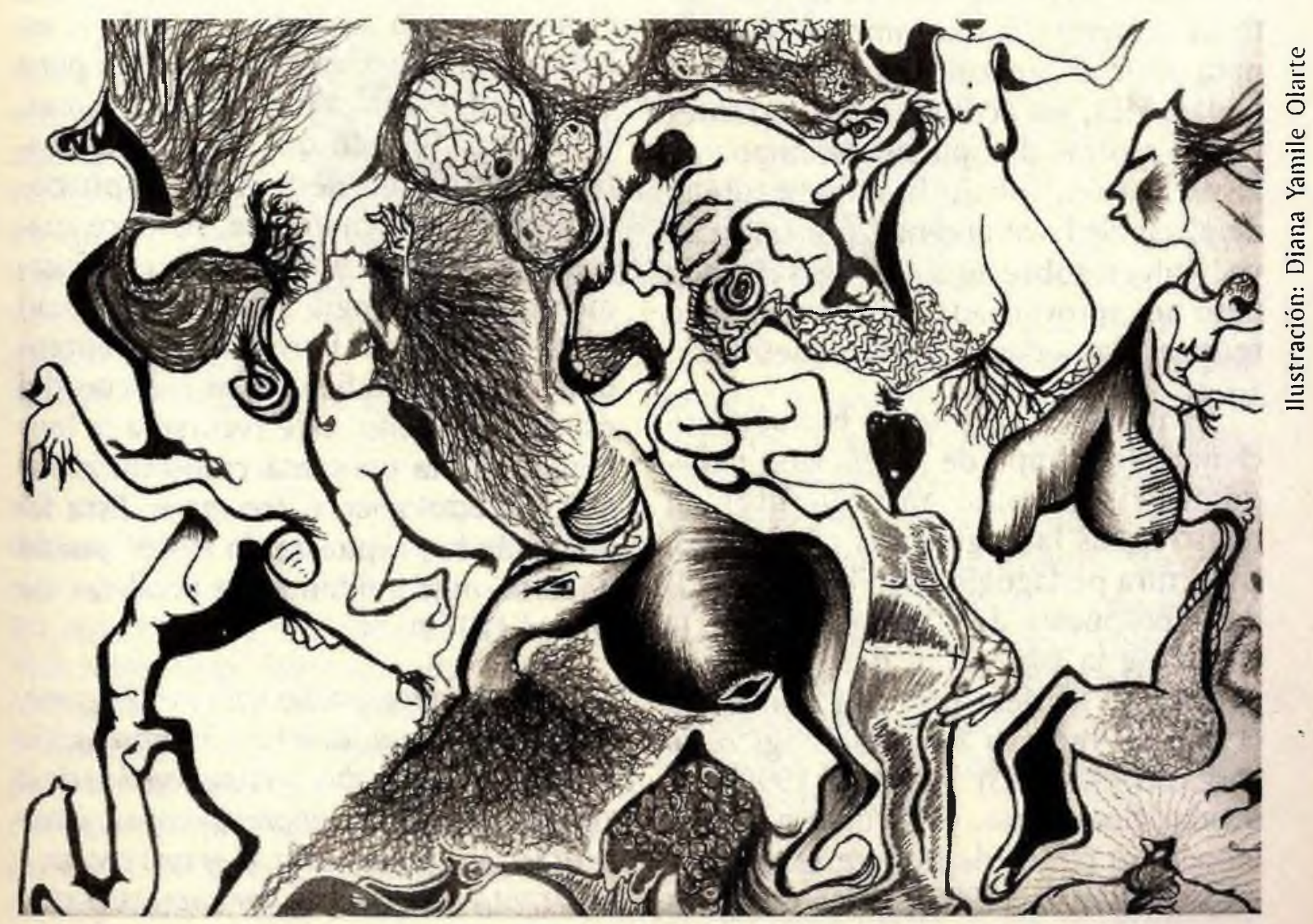


Universidad SuRcolombiana

esperado, una subordinación del discurso pedagógico y su campo de investigación, generalmente, reconfigurado desde discursos y campos con nuevas pretensiones hegemónicas, que ponen a la pedagogía en un permanente estado adámico. Inclusive, la misma propuesta del profesor Díaz para delimitar ese campo intelectual se enmarca, no en el contexto de la tradición pedagógica, sus conceptos y problemas, sino desde la sociolinguiística bernsteiniana (Bernstein 1990). La consecuencia negativa del "epistemismo", entonces, puede ser obviada si se ubica, no en una discusión estrictamente metodológica (cuantitativo-cualitativo), sino que se amplía el horizonte de las discusiones hacia la racionalidad y se opta, es la propuesta de esta contribución, por una visión ampliada hermeneúticamente del saber y el ser de la comprensión. Se pretende dotar al campo de la investigación pedagógica de un concepto de experiencia más amplio que permita abrir el horizonte de la reflexión pedagógica, sin actitudes vergonzantes frente a otras disciplinas o campos de investigación, o bajo la mirada tutelar de ellas. Se hace urgente, por tal motivo, volver sobre una vía ya señalada pero no aprovechada en su fecundidad teórica: la pedagogía hermeneútica.

El proyecto de ampliar hermenéuticamente el campo de la reflexión pedagógica tiene como antecedentes en nuestro país las siguientes propuestas: La lectura pedagógica de Flórez (1994) de la propuesta de Gadamer (1960), la visión de la educación desde la perspectiva de Ricoeur ofrecida por Rubio (1994), el recurso fenomenológico de Vargas (1992-1993) y Hoyos (1990), sin olvidar, finalmente, el recurso a la hermenéutica crítica de Habermas del grupo de Antanas (1995). Estas opciones comparten la perspectiva hermenéutica de muy diversas maneras, pero siempre en el contexto del "epistemismo" denunciado por Díaz, sin lograr, por tanto, que la pedagogía instaure un horizonte propio para la reflexión, la definición de una experiencia propia y la comprensión fecunda que permita ampliar el espectro de las indagaciones. Una presentación breve de cada una de las opciones ofrecerá una perspectiva de sus alcances y limitaciones.

Desde la publicación de "Pedagogía y verdad', el profesor Flórez (1989) propone la vinculación de la pedagogía con la hermeneútica, ya que hace posible remitir la pedagogía a las ciencias del espíritu y separarlas de las ciencias de la naturaleza. Este recurso a la hermenéutica adolece por principio de una actitud vergonzante, denunciada ya por Popper (1984) en "La miseria del historicismo" a saber, la búsqueda de un espacio propio de ciertas disciplinas en oposición a las ciencias naturales pero en clara desventaja frente a las mismas. Quizá sea por ello que, en la propuesta, el enfoque "deductivo-empíricoanalítico", implícitamente, siempre aparece como modélico cuando explícitamente la pedagogía se presenta como subsidiaria de la hermenéutica entendida como metódica de las ciencias del espíritu, por ello, este recurso a la hermenéutica la presenta como un enfoque metodológico entre otros. Esta falencia de la propuesta de Flórez puede hacerse más evidente en palabras de Heller (1989):

"No es necesario decir que la hermenéutica no es equivalente a interpretación por que es, de hecho, la comprensión de la comprensión, o la comprensión de la interpretación, y en tal calidad es una teoria, y por tanto explicativa. Por supuesto si se 
utiliza la hermenéutica no como teoría sino como método, y se hace tan concluyentemente, el resultado será una serie de interpretaciones textuales, una detrás de otras, sin ideas que guien, valores o metanarrativas de ningún tipo. Esta forma de mera interpretación, con carencia de teoría $y$ de explicación. origina una versión del positivismo incluso más estúpida y vacía..."

Sin embargo, frente a la menguada comprensión de la hermenéutica hecha por el autor, gana para la pedagogía conceptos como "historicidad", "tradición", "comprensión", "prejuicio" y "formación", que se convierten en alternativas frente a enfoques meramente cuantitativos en la investigación pedagógica o al paidocentrismo imperante.

Sólo hasta la publicación de " $\mathrm{Ha}$ cia una pedagogía del conocimiento" (Flórez 1994), el recurso a la hermenéutica se desplazó tímidamente del campo epistémico a una indagación histórica sobre la pedagogía como disciplina y se ubicó el concepto de formación como eje de la reflexión pedagógica, pero no se logra ahondar en las consecuencias de tal recurso, máxime cuando es obvio que el autor prefiere una concepción "cibernética" de la racio- nalidad que pretende hacer coincidir con la hermenéutica. Cabe anotar que, aún hoy, el autor continúa con su perspectiva menguada de la hermenéutica y, por ende, de la comprensión (Flórez 1998). Esta visión menguada se debe, quizá, a la inadecuada lectura hecha por Flórez del proyecto de Gadamer (1960) en Verdad y Método", quien a su vez, al abrir espacio para otras opciones frente al método de las ciencias empírico-analíticas, desvincula la comprensión del ser mismo como "Da sein", es decir, el comprender como método y no como condición misma del ser (Rühle 1991).

Rubio, propende en su recurso a la hermenéutica por ampliar la perspectiva crítica sobre la crisis de la educación contemporánea, definida en términos no de "problemas de formación científica" sino de relaciones entre culturas. Para el autor, "La revolución hermenéutica en la educación" busca "repolitizar la ciencia" vinculando la formación con las humanidades, ofreciendo una más estrecha relación entre educación y cultura en la clave del diálogo intercultural.

Vargas, desde su perspectiva fenomenológica, vuelve sobre la formación 
UNIVERSIDAD SURCOLOMBIANA

como "tema eje de la reflexión pedagógica" para establecer así una barrera al cientificismo y abrir caminos multidisciplinares en la investigación pedagógica. Hoyos, por su parte, considera de suma importancia ubicar la educación en general y la enseñanza en particular, como relación simbólica constructiva y crítica entre alumno-maestro, en la esfera de la razón comunicativa y evitar la colonización del "mundo de la vida" por la razón instrumental, instaurando en la pedagogía tres dimensiones: procesual, práctica y estratégica. Finalmente, el recurso a la hermenéutica crítica de Habermas por parte del grupo de Antanas, pretende definir la pedagogía como disciplina "reconstructiva", cuyo fin es reconstruir las "competencias" de los educadores y los alumnos, es decir, afrontar la transformación de un "saber-como" en un "saber que", en términos de "saber extrescolar" y "saber intraescolar".

Los esfuerzos anteriores no logran superar el "epistemismo" denunciado por Díaz, sobre todo por dos razones: en primer lugar, circunscriben el debate a una esfera exclusivamente metodológica y, por tanto, no logran vislumbrar una salida a la complejidad del espacio propio de las reflexiones pedagógicas, finalmente, no encuentran la manera de conciliar las "investigaciones de punta" con la cotidianidad de la enseñanza, es decir, la formación no alcanza a ofrecer el puente necesario para superar el mero activismo metodicista.

Retomando un camino ya abierto, y en clara oposición a las posturas escépticas (Gallego 1995 y Vasco 1995) frente a la pedagogía como una disciplina con su propio espacio para la reflexión, se ponen las siguientes tesis a consideración de los investigadores y maestros.

La pedagogía es la reflexión sobre diversas estrategias, generacionales $o$ institucionales, para la vinculación crítica del hombre con lo mejor de su tradición, lo que evita ver la tradición como un recurso conservador que desde la historia impide al sujeto nuevos cursos de acción desde un horizonte de expectativas $^{4}$ (Koselleck 1979), horizonte que abre espacio a la utopía, sin olvidar la historicidad de la experiencia humana. Incluye esta reflexión la validez y pertinencia de la selección y metódica presentación de tal tradición, en los diversos escenarios donde acaecen las múltiples interacciones entre sujetos desde la perspectiva de la formación.

La preocupación fundamental es comprender el proceso mediante el cual se apropia existencial y críticamente el hombre su tradición; no solamente como dato histórico en el cual se instala nuestro peculiar comprender, sino como espacio de experiencia y horizonte de expectativas; para develar aquellos presupuestos que están ya implícitos en cualquier estrategia institucional o generacional de vinculación con la tradición. No es exclusivamente una actividad de evaluación o proposición de métodos para mejorar el acceso a la tradición, se trata pues, de la reflexión, de un volver sobre la propia experiencia insistentemente para develar, hacer evidente, aquello que determina todo proceso de vinculación del hombre a

4. Los conceptos de "horizonte de expectativa" y "experiencia", son apropiados del arsenal argumental de la propuesta histórica de Koselleck. 
su tradición, todo proceso de formación, entendida ésta no como "un mero cultivo de disposiciones previas", sino como el proceso en el cual "uno se apropia por entero aquello en lo cual y a través de lo cual uno se forma" (Gadamer 1993) y expande su espacio de experiencia de sí y de los otros como horizonte de expectativas. En breves palabras, mantener el estado de abierto de la experiencia humana, no sometida ni al poder ni a la tradición como irrebasables.

La pedagogía se instala en ese segundo nivel reconocido ya por filósofos de las ciencias tradicionalmente reacios a asumir posiciones que los acercaran a la temida "especulación" 5 (Moulines 1995), es decir, la reflexión, en este caso pedagógica, que no excluye al maestro o lo subordina como simple reproductor de un discurso (Díaz 1993). La pedagogía no puede aceptar la diferenciación entre "experto" que orienta la acción y actor que sigue lineamientos, como si la acción pedagógica en la cotidianidad -puede ser del aula, por ejemplo- se puede efectuar sin reflexión alguna sobre los fines de tal acción y el contexto de su realización. En palabra del joven Benjamin (1994):

“.. La difusión de lo espiritual (y, en este sentido una cultura) constituye un problema. Es que, además, la difusión de lo espiritual supone una exigencia añadida: saber qué valores deseamos legar a la posteridad como la mejor de las herencias. La reforma escolar no es, pues, solo una reforma en la difusión de valores, sino que también representa una revisión de estos mismos valores".

La pedagogía no es, pues, una historiografía cultural o una historia de metas formativas y sus respectivas estrategias vinculantes, no es exclusivamente una detección y evaluación de registros para determinar la positividad del "discurso" pedagógico en un momento histórico, la pedagogía se ocupa de reflexionar sobre la historicidad misma de las estrategias vinculantes de la tradición sin renunciar al enjuiciamiento crítico de sus logros en el plano de la validez y pertinencia de la selección y metódica presentación de tal tradición, en el plano de la formación y el acceso a la condición humana. No sobra decir que la pedagogía adquiere así un carácter antropológico y ético, es decir, no es preceptiva metódica sino una reflexión crítica de los fines formativos, se instala en la esfera propia de lo práctico, para expresarlo en términos kantianos.

Tampoco es la pedagogía una creación constante de metódicas para optimizar la vinculación con ciertos saberes de la tradición, como si en el mero contenido de saberes fragmentarios se definiera la condición humana misma, manifestación de una estrechez de miras en el análisis del rol de las ciencias en la existencia pluriforme del

5. Aunque Molines concibe como meramente "gramatical" el posible recurso a la hermenéutica, es rescatable el reconocimiento del segundo nivel en que se instala la filosofía de las ciencias llamado por el autor "fenómeno recursivo", es decir, "teorización de teorizaciones" o "interpretación de interpretaciones". Lo anterior no es otra cosa que la reflexión, en principio. 


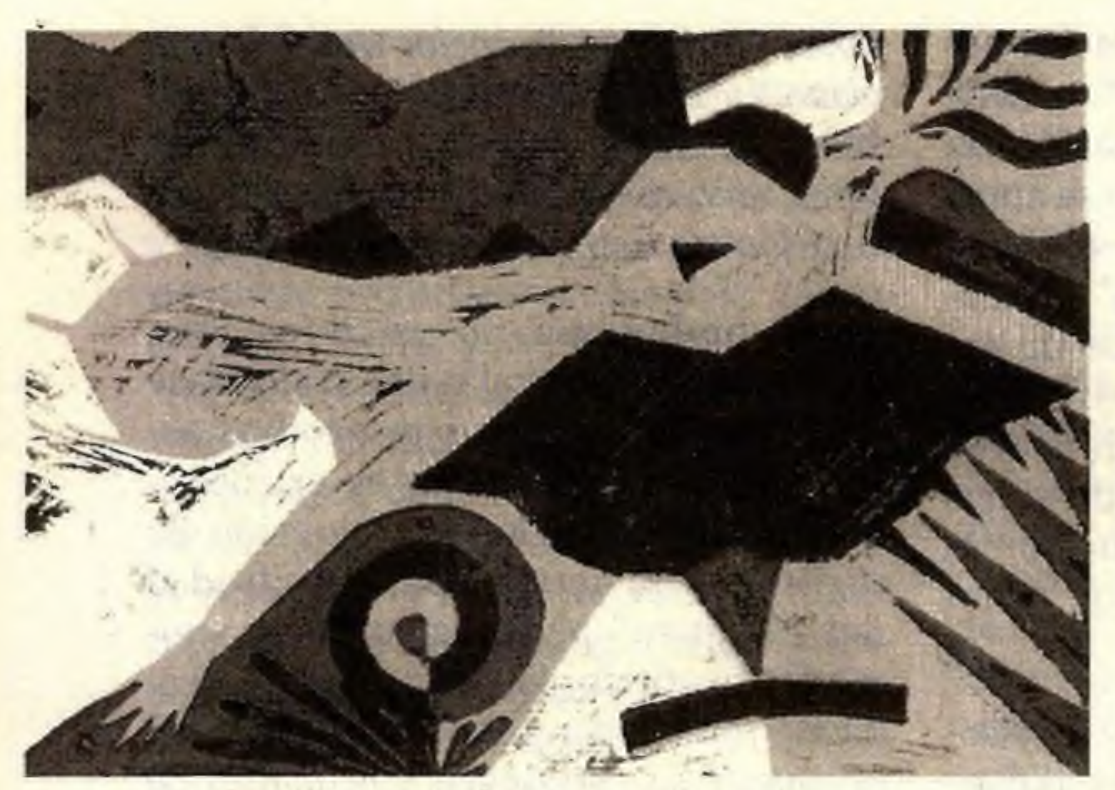

Ilustración: Alexander Salazar H.

de los espacios de sentido más privilegiados por la tradición pedagógica donde se opera la vinculación crítica del hombre con lo mejor de su tradición. La reflexión pedagógica, ineludible, es la posibilidad de ofrecer unidad -siempre abierta- de objeto y método a las indagaciones educativas que hasta ahora se mantienen en dos planos: la perspectiva de los especialistas (especuladores o experimentadores) y la cotidianidad de la enseñanza.

hombre. En este sentido, confundir la pedagogía con la exploración sicológica o neuronal de aquello que acontece en uno, y solo uno, de los procesos acaecidos en la formación no es correcto, máxime cuando desde posiciones tales se pretende eludir, bajo la acusación de ser mera especulación, la reflexión propia de la pedagogía. La pedagogía, para no continuar con el tono de teología negativa, explora esencialmente la donación de sentido acaecida en la formación con todo el amplio espectro de la reflexión que ello implica; se ocupa de comprender las estrategias de vinculación con la tradición, de abrirse a sus consecuencias prácticas y estar alerta frente a los dogmatismos y reducciones de las posibilidades del hombre de comprender su tradición y abrirse a nuevos horizontes de expectativa.

La pedagogía no es preceptiva metódica, se ocupa de los fines formativos, no se agota en la relación maestro-alumno, ni aún en la mediación lingüística entre ambos, como proponen las pedagogías comunicativas. Tampoco se agota en el aula, aunque es uno
En definitiva, la pedagogía estará abierta a los aportes de las disciplinas que hasta ahora reclaman su hegemonía, en lo que se conoce como "ciencias de la educación", entre ellas la historiografía, la sicología desde sus aportes a la metódica, pero no aceptará ser reconfigurada exclusivamente por una de ellas. Esta condición de apertura de la pedagogía hará más fecunda las indagaciones y permitirá trascender así lo estrictamente coyuntural y político, en lo cual se confunde el proyecto formativo con aquellas condiciones materiales para su realización, donde prima una racionalidad económica, a caballo siempre entre la novedad y la superficialidad acrítica. Sólo nos vinculamos a la pedagogía si nuestras investigaciones y su lenguaje dialogan con la tradición pedagógica como contexto y referente propio donde se legitiman los conceptos, los métodos y las experiencias, en pocas palabras: estamos abocados a la historia conceptual de la reflexión pedagógica para avanzar en nuestras investigaciones con sentido. 
PAIDEIA

\section{Referencias Bibliográficas}

BENJAMIN, Walter. (1912) La reforma escolar: un movimiento cultural. Barcelona, Altea, 1994. pp 47-52.

BERNSTEIN, Basil. (1990) La estructura del discurso pedagógico. Madrid, Morata, 1994. pp. 236.

DÍAZ, Mario. (1993) El campo intelectual de la educación en Colombia. Santiago de Cali, Universidad del Valle, 263 pp.

FLÓREZ, Rafael. (1994) Hacia una pedagogía del conocimiento. Santafé de Bogotá, McGrawHill. $311 \mathrm{pp}$.

FLÓREZ, Rafael. (1989) Pedagogía y Verdad. Medellín, Secretaría de Educación y Cultura Departamental. 235 pp.

FLÓREZ, Rafael. (1998) El fin de la historia o el fin de la pedagogía. En: Cuadernos Pedagógicos, Medellín, No. 2. pp 17 y ss.

GADAMER, Hans Georg. (1960) Verdad y método. Salamanca, Sígueme, 1993. 697 pp.

GALLEGO B, Rómulo. (1995) Saber pedagógico, una visión alternativa. Santafé de Bogotá, Magisterio, $170 \mathrm{pp}$.

HELLER, Agnes. (1989) De la hermenéutica en las ciencias sociales a la hermenéutica en las ciencias sociales. En: FEHER, Ferenc. Políticas de la posmodernidad, ensayos de crítica cultural. Barcelona, Península. pp 52 y ss.

HOYOS V, Guillermo. (1990) Prólogo. En: DİAZ, Mario y MUÑOZ, José. Pedagogía, discurso y poder. Bogotá, CORPRODIC. PP 13 Y SS.

KOSELLECK, Reinhart. (1979) Pasado futuro, para una semántica de los tiempos históricos. Barcelona, Paidós, 1993. pp 333 y ss.

MOCKUS, Antanas y otros. (1995) Las fronteras de la escuela. Santafé de Bogotá, Magisterio. 129 pp.

MOULINES, Ulises. (1995) La filosofia de la ciencia como disciplina hermenéutica. En: Isegoria, Madrid, No. 12, octubre.

POPPER, Karl R. (1984) La miseria del historicismo. Madrid, Alianza-Taurus. $176 \mathrm{pp}$.

RUBIO A, Jaime. (1994) Un proyecto hermenéutico para la educación contemporánea. Santafé de Bogotá, Universidad Pontificia Javeriana. $68 \mathrm{pp}$.

RÜHLE, Volker. (1991) Experiencia de la historia y experiencia histórica, sobre el concepto de conciencia histórico efectual en Gadamer. En: Isegoría, Madrid. No. 4 octubre.

SOKAL, Allan. (1998) Transgrediendo Los límites: hacia una hermenéutica transformadora de la gravedad cuántica. En: la balsa de la medusa. Madrid. No, 45-46 


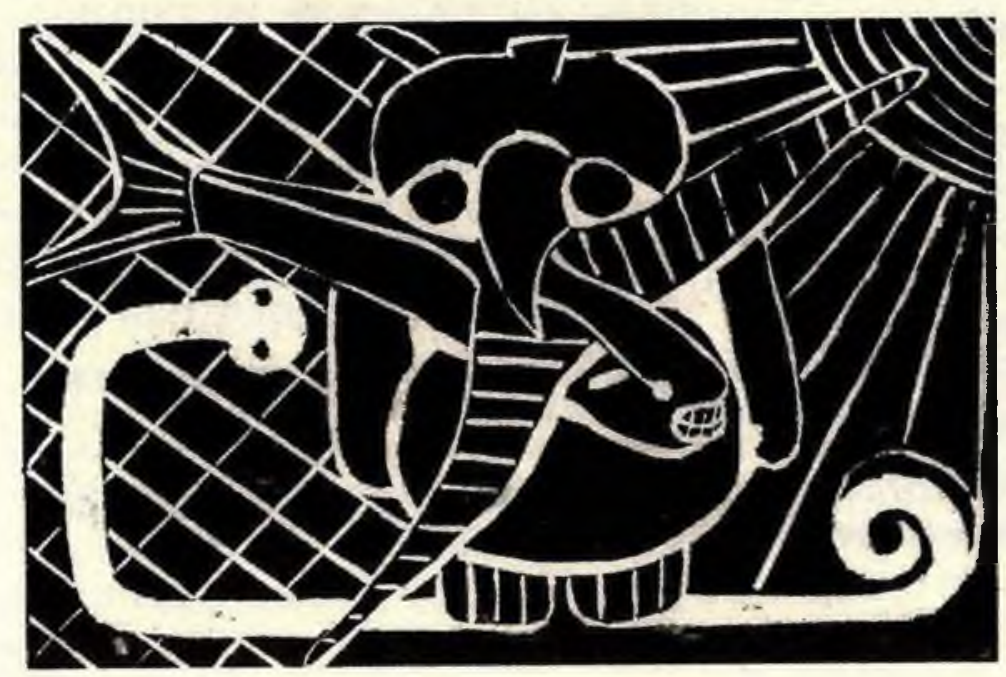

Ilustración: Alexander Salazar H. 